\title{
Covering a reduced spherical body by a disk
}

\author{
Michał Musielak
}

\begin{abstract}
In this paper, the following two theorems are proved: (1) every spherical convex body $W$ of constant width $\Delta(W) \geq \frac{\pi}{2}$ may be covered by a disk of radius $\Delta(W)+\arcsin \left(\frac{2 \sqrt{3}}{3} \cdot \cos \frac{\Delta(W)}{2}\right)-\frac{\pi}{2} ;(2)$ every reduced spherical convex body $R$ of thickness $\Delta(R)<\frac{\pi}{2}$ may be covered by a disk of radius $\arctan \left(\sqrt{2} \cdot \tan \frac{\Delta(R)}{2}\right)$.
\end{abstract}

Mathematics Subject Classification (2010). Primary 52A55.

Keywords. spherical convex body, spherical geometry, hemisphere, lune, width, thickness, disk.

\section{Introduction}

The subject of reduced bodies in the Euclidean space $E^{d}$ has been well researched, see for instance [6]. However, it is a natural idea to investigate such bodies in non-Euclidean geometries. There are number of articles describing reduced bodies on the sphere, see: [5], 3], [7, [8. The question arises which of the results achieved in the Euclidean space can be transferred to the sphere. In particular, the main theorem of this paper presents the spherical version of the variant of Jung theorem for reduced bodies in $E^{2}$ given in [4].

Let $S^{2}$ be the unit sphere of the three-dimensional Euclidean space $E^{3}$. By a great circle of $S^{2}$ we mean the intersection of $S^{2}$ with any twodimensional subspace of $E^{3}$. The set of points of a great circle of $S^{2}$ in the distance at most $\frac{\pi}{2}$ from a point $c$ of this great circle is called a semicircle with center $c$. Any pair of points obtained as the intersection of $S^{2}$ with a one-dimensional subspace of $E^{3}$ is called a pair of antipodes. Note that if two different points $a, b$ are not antipodes, there is exactly one great circle containing them. The shorter part of this great circle is called the spherical arc connecting $a$ and $b$, or shortly arc. It is denoted $a b$. By the spherical distance $|a b|$, or shortly distance, of these points we mean the length of the arc $a b$. If $a, b$ are antipodes, we put $|a b|=\pi$. If $p$ is a point of $S^{2}$ and $F$ 
is a closed set containing at least two points, then we $\operatorname{define} \operatorname{dist}(p, F)$ as $\min _{q \in F}|p q|$.

A subset of $S^{2}$ is called convex if it does not contain any pair of antipodes of $S^{2}$ and if together with every two points it contains the arc connecting them. By a spherical convex body we mean a closed convex set with nonempty interior. If there is no arc in the boundary of a spherical convex body, we call the body strictly convex.

Let $\rho \in\left(0, \frac{\pi}{2}\right]$. By disk of radius $\rho$ and center $c$ we mean the set of points of $S^{2}$ in the distance at most $\rho$ from a point $c \in S^{2}$. The boundary of a disk is called a spherical circle. By a hemisphere we mean any disk of radius $\frac{\pi}{2}$. The hemisphere with center $p$ is denoted by $H(p)$. If $p, q$ is are antipodes, then $H(p)$ and $H(q)$ are called opposite hemispheres. Let $t$ be a boundary point of a convex body $C \subset S^{2}$. We say that a hemisphere $H$ supports $C$ at $t$ if $C \subset H$ and $t$ belongs to the great circle bounding $H$. If the body $C$ is supported at $p$ by exactly one hemisphere, we call $p$ a smooth point of the boundary of $C$. If all boundary points of $C$ are smooth, we say that $C$ is smooth. We say that $e$ is an extreme point of $C$ if $C \backslash\{e\}$ is a convex set. If the set $A$ is contained in an open hemisphere, we define $\operatorname{conv}(A)$ as a smallest convex set containing $A$ (for details see definition before Lemma 1 in [5])

If hemispheres $G$ and $H$ are different and not opposite, then $L=G \cap H$ is called a lune. The two semicircles bounding $L$ and contained in $G$ and $H$, respectively, are denoted by $G / H$ and $H / G$. The thickness $\Delta(L)$ of $L \subset S^{2}$ is defined as the distance of the centers of $G / H$ and $H / G$. By the corners of $L$ we understand the two points of the set $(G / H) \cap(H / G)$.

For every hemisphere $K$ supporting a convex body $C \subset S^{2}$ we find hemispheres $K^{*}$ supporting $C$ such that the lunes $K \cap K^{*}$ are of the minimum thickness (by compactness arguments at least one such a hemisphere $K^{*}$ exists). The thickness of the lune $K \cap K^{*}$ is called the width of $C$ determined by $K$ and it is denoted by width $K(C)$ (see $[5]$ ). If for all hemispheres $K$ supporting $C$ the numbers width $_{K}(C)$ are equal, we say that $C$ is of constant width (see [5]). By the thickness $\Delta(C)$ of a convex body $C \subset S^{2}$ we understand the minimum width of $C$ determined by $K$ over all supporting hemispheres $K$ of $C$ (see [5]).

After [5] we call a spherical convex body $R \subset S^{2}$ reduced if $\Delta(Z)<$ $\Delta(R)$ for every convex body $Z \subset R$ different from $R$. Simple examples of reduced spherical convex bodies on $S^{2}$ are spherical bodies of constant width and, in particular, the disks on $S^{2}$. Also each of the four parts of a spherical disk on $S^{2}$ dissected by two orthogonal great circles through the center of the disk is a reduced spherical body. It is called a quarter of a spherical disk.

Note: most of the above notions can be defined also in higher dimensions, for details see for instance [5].

For the convenience of the reader recall a few formulas of spherical geometry which are frequently applied in this paper. Consider the right spherical triangle with hypotenuse $C$ and $\operatorname{legs} A, B$. Denote by $\alpha$ the angle opposite to 
$A$ and by $\beta$ the angle opposite to $B$. By [11] the following formulas hold true

$$
\begin{aligned}
\tan A & =\cos \beta \tan C \\
\sin A & =\sin \alpha \sin C \\
\cos C & =\cos A \cos B \\
\cos C & =\cot \alpha \cot \beta .
\end{aligned}
$$

After 2] we define the circumradius of a convex body $C$ as the smallest $\rho$ such that $C$ can be covered by a disk of radius $\rho$. Such a disk is unique and is called the disk circumscribed about $C$. The boundary of this disk is called the circle circumscribed about $C$. In particular the circle circumsribed on a spherical triangle contains all vertices of this triangle, which is a consequence of the spherical Ceva's theorem (see: Theorem 2 of [9]).

Determining the circumradius in the three special cases presented in the below Lemmas 1, 3 is useful in the further part of the paper.

Lemma 1. Let $Q \subset S^{2}$ be a quarter of disk. The circumradius of $Q$ is equal $\rho=\arctan \left(\sqrt{2} \cdot \tan \frac{\Delta(Q)}{2}\right)$.

Proof. Denote by $c$ the center of the disk whose quarter is considered and by $a, b$ the two different extreme points of $Q$ such that $c a$ and $c b$ are subsets of the boundary of $Q$. It is easily seen that if a disk contains points $a, b, c$, then it contains $Q$. Therefore we are looking for the radius $\rho$ of the disk circumscribed on the triangle $a b c$. Denote by $o$ the center of this disk and by $p$ a point on $a c$ such that the angle $\angle c p o$ is right. Since $o$ is in equal distances from $a$ and $c$, the point $p$ is in the middle of $a c$ and thus $|c p|=\frac{\Delta(Q)}{2}$. Clearly, the angle $\angle p c o$ equals $\frac{\pi}{4}$ and $|o c|$ is equal to the radius of our disk. Hence, considering the triangle $\operatorname{cop}$, by (11) we have $\tan \frac{\Delta(Q)}{2}=\cos \frac{\pi}{4} \tan \rho$. By evaluating $\rho$ we obtain the thesis of our lemma.

Recall after [5] that Reuleaux triangle is the intersection of three disks of radius $\sigma$ such that the centers of these disks are pairwise distant by $\sigma$.

Lemma 2. The circumradius of a spherical Reuleaux triangle $R$ is equal $\rho=\arcsin \left(\frac{2 \sqrt{3}}{3} \cdot \sin \frac{\Delta(R)}{2}\right)$.

Proof. Denote by $a, b, c$ the three points of $\operatorname{bd}(R)$ that are not smooth. It is easy to check that any disk containing these three points contains $R$. Thus $\rho$ is equal to the radius of the disk circumscribed on the triangle $a b c$. Denote by $o$ the center of this disk and by $p$ the middle of the arc $a b$. Clearly, the angle $\angle o p a$ is right, the angle $\angle a o p$ is equal $\frac{\pi}{3}$ and $|a p|=\frac{\Delta(R)}{2}$. Therefore, if we look at the triangle $a o p$, by (2) we obtain $\sin \frac{\Delta(R)}{2}=\sin \frac{\pi}{3} \sin \rho$. We easily evaluate $\rho$ which establishes the promised formula.

Lemma 3. The circumradius $\rho$ of a spherical equilateral triangle $T$ of thickness less than $\frac{\pi}{2}$ equals arctan $\frac{\sqrt{9+8 \tan ^{2} \Delta(T)}-3}{2 \tan \Delta(T)}$. 
Proof. Denote by o the center of the disk circumscribed on our triangle, by $d$ the center of a side of this triangle and by $a$ an endpoint of this side. Clearly $\angle d o a=\frac{\pi}{3},|o a|=\rho$ and $|o d|=\Delta(T)-\rho$. Therefore by (11) we obtain $\tan (\Delta(T)-\rho)=\frac{1}{2} \tan \rho$. Using the subtraction formula for the tangent function we can rewrite this equation as $\frac{\tan \Delta(T)-\tan \rho}{1+\tan \Delta(T) \tan \rho}=\frac{\tan \rho}{2}$. This is equivalent to $\tan \Delta(T)(\tan \rho)^{2}+3 \tan \rho-2 \tan \Delta(T)=0$. Consequently, by $\tan \rho>0$ we get $\tan \rho=\frac{\sqrt{9+8 \tan ^{2} \Delta(T)}-3}{2 \tan \Delta(T)}$, which ends the proof.

\section{Covering a body of constant width over $\frac{\pi}{2}$ by a disk}

For any set $F$ on the sphere $S^{2}$ we define the set $F^{\oplus}$ as $\{p: F \subset H(p)\}$.

In [3] and [12] there is used the notion of the polar set of the set $F$ on the sphere, which is defined as $F^{o}=\bigcap_{p \in F} H(p)$.

Proposition 1. For every set $F$ on the sphere we have $F^{o}=F^{\oplus}$.

Proof. For any point $q$ we have: $q \in F^{o} \Leftrightarrow \forall_{p \in F} q \in H(p) \Leftrightarrow \forall_{p \in F}|p q| \leq$ $\frac{\pi}{2} \Leftrightarrow \forall_{p \in F} p \in H(q) \Leftrightarrow F \subset H(q) \Leftrightarrow q \in F^{\oplus}$.

However, applying $F^{\oplus}$ is more convenient in this paper.

We omit here a simple proof of the next lemma

Lemma 4. If $C$ is a spherical convex body, then $C^{\oplus}$ is also a spherical convex body.

By the way, observe that $\left(C^{\oplus}\right)^{\oplus}=C$ for a spherical convex body $C$.

Proposition 2. If $W$ is a spherical convex body of constant width, then $W^{\oplus}$ is a spherical convex body of constant width $\pi-\Delta(W)$.

Proof. Consider a hemisphere $H(a)$ supporting $W^{\oplus}$. We intend to show that width $_{H(a)}\left(W^{\oplus}\right)=\pi-\Delta(W)$. Since clearly $a$ is a boundary point of $W$, by Theorem 7 of [7] there exist hemispheres $K$ and $M$ supporting $W$ such that the lune $K \cap M$ is of thickness $\Delta(W)$ and $a$ is the center of $K / M$. Denote the center of the semicircle $M / K$ by $b$ and the centers of the hemispheres $K$ and $M$ by $a^{\prime}$ and $b^{\prime}$, respectively. Since $a, b, a^{\prime}, b^{\prime}$ are in the same distance from both corners of $K \cap M$, all these points lay on the same great circle. From the above we easily obtain that $|a b|+\left|a^{\prime} b^{\prime}\right|=\left|a a^{\prime}\right|+\left|b b^{\prime}\right|=\pi$ and thus $\left|a^{\prime} b^{\prime}\right|=\pi-\Delta(W)$. Since every point of $W^{\oplus}$ is in the distance at most $\frac{\pi}{2}$ from $b$, the body $W^{\oplus}$ is contained in $H(b)$. Therefore $W^{\oplus}$ is contained in the lune $H(a) \cap H(b)$, which is of thickness $\left|a^{\prime} b^{\prime}\right|$. It means that width ${ }_{H(a)}\left(W^{\oplus}\right)$ is at most $\pi-\Delta(W)$.

Assume that width ${ }_{H(a)}\left(W^{\oplus}\right)<\pi-\Delta(W)$. Then there exists a point $\bar{b}$ such that the lune $H(a) \cap H(\bar{b})$ is of thickness less that $\pi-\Delta(W)$ and contains $W^{\oplus}$. From $|a \bar{b}|+\Delta(H(a) \cap H(\bar{b}))=\pi$ we conclude that $|a \bar{b}|>\Delta(W)$. But 
this contradicts the fact that every body $W$ of constant width has diameter $\Delta(W)$. Hence, width $_{H(a)}\left(W^{\oplus}\right)=\pi-\Delta(W)$, which ends the proof.

In 2] there was estimated the diameter of a spherical compact set by the function of its circumradius (see: the second part of Theorem 2 in 2]). Recall this result on $S^{2}$ in a different form: if $d$ is the diameter of a compact set and $\sigma$ is its circumradius, then $\sin \sigma \leq \frac{2 \sqrt{3}}{3} \cdot \sin \frac{d}{2}$. In particular it holds true for every spherical convex body $W$ of constant width and in this case $d=\Delta(W)$. If $\Delta(W)$ is at most $\frac{2 \pi}{3}$ then $\frac{2 \sqrt{3}}{3} \cdot \sin \frac{d}{2}$ is at most 1 . In this case our inequality is equivalent to the statement that every spherical convex body of constant width at most $\frac{2 \pi}{3}$ can be covered by a disk of radius $\arcsin \left(\frac{2 \sqrt{3}}{3} \cdot \sin \frac{\Delta(W)}{2}\right)$. If $\Delta(W)$ is greater than $\frac{2 \pi}{3}$ then $\frac{2 \sqrt{3}}{3} \cdot \sin \frac{d}{2}$ is greater than 1 and in this case our inequality does not estimate $\sigma$ in a non-trivial way.

According to Lemma 2 the example of a spherical Reuleaux triangle shows that the estimate can not be improved for bodies of constant width at most $\frac{\pi}{2}$. The following theorem describes the case of bodies of constant width at least $\frac{\pi}{2}$ and in particular gives an improvement of the estimate recalled from [2] for convex bodies of constant width greater than $\frac{\pi}{2}$.

Theorem 1. Every spherical body $W$ of constant width at least $\frac{\pi}{2}$ is contained in a disk of radius $\Delta(W)+\arcsin \left(\frac{2 \sqrt{3}}{3} \cdot \cos \frac{\Delta(W)}{2}\right)-\frac{\pi}{2}$.

Proof. Observe that for $\Delta(W)=\frac{\pi}{2}$ we have $\arcsin \left(\frac{2 \sqrt{3}}{3} \cdot \cos \frac{\Delta(W)}{2}\right)+$ $\Delta(W)-\frac{\pi}{2}=\arcsin \left(\frac{2 \sqrt{3}}{3} \cdot \sin \frac{\Delta(W)}{2}\right)$. Therefore by the above recalled result of Dekster the thesis of the theorem holds true for bodies of constant width $\frac{\pi}{2}$.

Assume now that $\Delta(W)>\frac{\pi}{2}$. By Proposition 2 the body $W^{\oplus}$ is of thickness $\pi-\Delta(W)$. Hence by the above recalled result of Dekster, $W^{\oplus}$ is contained in a disk of radius $\arcsin \left(\frac{2 \sqrt{3}}{3} \cdot \sin \frac{\pi-\Delta(W)}{2}\right)=$ $\arcsin \left(\frac{2 \sqrt{3}}{3} \cdot \cos \frac{\Delta(W)}{2}\right)$. Denote the center of this disk by $o$ and let $p$ be a boundary point of $W$. By Proposition 3 of [7] $W$ is smooth, and therefore there exists exactly one hemisphere supporting $W$ at $p$. Denote its center by $a$ and notice that $a$ is a boundary point of $W^{\oplus}$. Moreover, $H(p)$ is a supporting hemisphere of $W^{\oplus}$ and it supports $W^{\oplus}$ at $a$. By the proof of the first part of Theorem 1 of [5], there exists a unique point of $W^{\oplus}$ closest to $p$. Denote it by $b$. Again by Theorem 1 of [5] $b$ is the center of one of the two semicircles bounding the lune of thickness width w $_{(p)}\left(W^{\oplus}\right)=\Delta\left(W^{\oplus}\right)=$ $\pi-\Delta(W)$. Thus $|a b|=\Delta\left(W^{\oplus}\right)$ and clearly $b \in a p$. Hence $|o p| \leq|o b|+|b p|=$ 
$|o b|+|a p|-|a b|=|o b|+\frac{\pi}{2}-(\pi-\Delta(W))=|o b|+\Delta(W)-\frac{\pi}{2}$. Since $|o b|$ is at most $\arcsin \left(\frac{2 \sqrt{3}}{3} \cdot \cos \frac{\Delta(W)}{2}\right)$, the distance between $o$ and $p$ is at most $\arcsin \left(\frac{2 \sqrt{3}}{3} \cdot \cos \frac{\Delta(W)}{2}\right)+\Delta(W)-\frac{\pi}{2}$ which ends the proof.

Observe that in general we can not improve the estimate from Theorem 1. By proof of this theorem we see that the value $\Delta(W)+\arcsin \left(\frac{2 \sqrt{3}}{3} \cdot \cos \frac{\Delta(W)}{2}\right)-$ $\frac{\pi}{2}$ is attained for every $W$ such that $W^{\oplus}$ is a Reuleaux triangle.

\section{Covering reduced bodies of thickness at most $\frac{\pi}{2}$ by a disk}

The main theorem of this paper is analogous to Theorem of [4. However, we are not able to present a similar proof as in [4] due to the lack of the notion parallelism on the sphere. For this reason the proof of our main theorem is based on a different idea.

Lemma 5. Let $c$ be a positive number less than $\frac{1}{4}$ and a be a number from the interval $\left(\frac{1}{2}, \frac{1}{2}+\sqrt{\frac{1}{4}-c}\right)$. The function $f(x)=\sqrt{1-x}-\sqrt{1-x-\frac{c}{x}}$ satisfies $f(x) \leq \max \left(f\left(\frac{1}{2}\right), f(a)\right)$ for every $x \in\left[\frac{1}{2}, a\right]$.

Proof. Notice that if $x>0$, then $1-x-\frac{c}{x} \geq 0$ is equivalent to $x^{2}-x+c \leq 0$. This inequality is satisfied for $x \in\left[\frac{1}{2}-\sqrt{\frac{1}{4}-c}, \frac{1}{2}+\sqrt{\frac{1}{4}-c}\right]$. In particular we conclude that $\sqrt{1-x-\frac{c}{x}}=0$ for $x=\frac{1}{2}+\sqrt{\frac{1}{4}-c}$ and $f(x)$ is well defined in the interval $\left(\frac{1}{2}, \frac{1}{2}+\sqrt{\frac{1}{4}-c}\right)$

In order to prove the thesis we check the sign of the first derivative of $f(x)$. We have $f^{\prime}(x)=-\frac{1}{2 \sqrt{1-x}}-\frac{1}{2 \sqrt{1-x-\frac{c}{x}}} \cdot\left(-1+\frac{c}{x^{2}}\right)=\frac{1}{2 \sqrt{1-x-\frac{c}{x}}}$ $\left(1-\frac{c}{x^{2}}-\sqrt{1-\frac{c}{x(1-x)}}\right)$.

Put $g(x)=1-\frac{c}{x^{2}}-\sqrt{1-\frac{c}{x(1-x)}}$. Clearly, for every $x$ the sign of $f^{\prime}(x)$ is the same as the sign of $g(x)$. We have $g(x)=0 \Leftrightarrow 1-\frac{c}{x^{2}}=\sqrt{1-\frac{c}{x(1-x)}} \Leftrightarrow$ $1-\frac{2 c}{x^{2}}+\frac{c^{2}}{x^{4}}=1-\frac{c}{x(1-x)} \Leftrightarrow \frac{1}{1-x}-\frac{2}{x}+\frac{c}{x^{3}}=0 \Leftrightarrow 3 x^{3}-2 x^{2}+c(1-x)=0$.

For $V(x)=3 x^{3}-2 x^{2}+c(1-x)$ we have $V(0)=c>0$ and $V\left(\frac{1}{2}\right)=$ $\frac{1}{2}\left(c-\frac{1}{4}\right)<0$. Thus $V(x)$ has three zeros: one is less than 0 , one is in the interval $\left(0, \frac{1}{2}\right)$, and one is greater than $\frac{1}{2}$. Hence, $g(x)$ has exactly one zero in the interval $\left(\frac{1}{2}, \frac{1}{2}+\sqrt{\frac{1}{4}-c}\right)$. Denote it by $x_{0}$. Due to the continuity of the function $g(x)$ in the interval $\left(\frac{1}{2}, \frac{1}{2}+\sqrt{\frac{1}{4}-c}\right)$, it has constant sign in the interval $\left(\frac{1}{2}, x_{0}\right)$ and constant sign in the interval $\left(x_{0}, \frac{1}{2}+\sqrt{\frac{1}{4}-c}\right)$. 
Notice that $g\left(\frac{1}{2}\right)=1-4 c-\sqrt{1-4 c}=(1-4 c)(\sqrt{1-4 c}-1)<0$ and $g\left(\frac{1}{2}+\sqrt{\frac{1}{4}-c}\right)=\sqrt{\frac{1}{2}-\sqrt{\frac{1}{4}-c}}>0$. Hence, $g(x)<0$ for $x \in\left(\frac{1}{2}, x_{0}\right)$ and $g(x)>0$ for $x \in\left(x_{0}, a\right)$. Therefore $f(x)$ is decreasing in $\left(\frac{1}{2}, x_{0}\right)$ and increasing in $\left(x_{0}, a\right)$. The thesis of our lemma is an immediate consequence of this statement.

Lemma 6. Let $a, b$ be points on a spherical circle. Consider any point $t$ such that $a, t, b$ lay on this circle in this order according to the positive orientation. The measure of the angle $\angle a t b$ is the greatest if $t$ is equidistant from a and $b$.

Proof. Denote by $o$ the center of our circle and by $\rho$ its radius. Denote by $t^{\prime}$ the point on our circle laying in equal distances from $a$ and $b$, and on the same side of the great circle containing $a b$ as $t$. Put $\left.\alpha=\frac{1}{2}|\angle a o t|\right]$ and $\beta=\frac{1}{2}|\angle b o t|=$, and let $k$ be the midpoint of the arc at. Observe that $\left|\angle a o t^{\prime}\right|=$ $\left|\angle b o t^{\prime}\right|=\alpha+\beta$. Since $|\angle t o k|=\alpha$, applying (4) we easily obtain that $|\angle o t a|=$ $\operatorname{arccot}(\cos \rho \tan \alpha)$. Analogously $|\angle o t b|=\operatorname{arccot}(\cos \rho \tan \beta)$ and $\left|\angle o t^{\prime} a\right|=$ $\left|\angle o t^{\prime} b\right|=\operatorname{arccot}\left(\cos \rho \tan \frac{\alpha+\beta}{2}\right)$. Our aim is to show that $\left|\angle a t^{\prime} b\right| \geq|\angle a t b|$.

This inequality is equivalent to $\operatorname{arccot}\left(\cos \rho \tan \frac{\alpha+\beta}{2}\right) \geq$ $\frac{\operatorname{arccot}(\cos \rho \tan \alpha)+\operatorname{arccot}(\cos \rho \tan \beta)}{2}$. In order to show this it is sufficient to show that the function $f(x)=\operatorname{arccot}(\cos \rho \tan x)$ is concave in the interval $\left(0, \frac{\pi}{2}\right)$. The reader may check that $f^{\prime \prime}(x)=\frac{2 \cos \rho \sin x \cos x\left(\cos ^{2} \rho-1\right)}{\left(\cos ^{2} x+\cos ^{2} \rho \sin ^{2} x\right)^{2}}$.

It is easily seen that $f^{\prime \prime}(x)<0$ for $x \in\left(0, \frac{\pi}{2}\right)$ and therefore the function $f(x)$ is concave in the interval $\left(0, \frac{\pi}{2}\right)$ which completes the proof.

Theorem 2. Every reduced spherical body $R$ of thickness at most $\frac{\pi}{2}$ is contained in a disk of radius $\rho=\arctan \left(\sqrt{2} \cdot \tan \frac{\Delta(R)}{2}\right)$.

Proof. Note that every boundary point of $R$ belongs to an arc whose ends are extreme points of $R$. Therefore if a disk contains all extreme points of $R$, then it contains all boundary points of $R$ and so the whole body $R$. Thus, it is sufficient to show that all extreme points of $R$ are in a disk of radius $\rho$. Moreover, according to the spherical Helly's Theorem (see: 10] and [1]), it is even sufficient to show that every three extreme points of $R$ are contained in a disk of radius $\rho$.

Let $e_{1}, e_{2}, e_{3}$ be any three different extreme points of $R$. By Theorem 4 of [5] there exist lunes $L_{1}, L_{2}, L_{3}$ such that $e_{i}$ is the center of one of the semicircles bounding $L_{i}$ for $i=1,2,3$. Denote by $f_{i}$ the center of the other semicircle bounding $L_{i}$ for $i=1,2,3$.

First let us consider the case when two points from amongst $e_{1}, e_{2}, e_{3}$, say $e_{1}$ and $e_{2}$, lay in $L_{3}$ on the same side of the great circle containing $e_{3}$ 
and $f_{3}$. Since $e_{1} f_{1}$ and $e_{3} f_{3}$ intersect (see the proof of Lemma 2 in [7]), the distance from $e_{1}$ to $e_{3} f_{3}$ is less or equal than the distance from $e_{1}$ to the point of intersection of $e_{1} f_{1}$ with $e_{3} f_{3}$, and so it is less or equal $\Delta(R)$. For the same reason the distance from $e_{2}$ to $e_{3} f_{3}$ is at most $\Delta(R)$. Denote by $c$ the corner of $L_{3}$ laying on the same side of the great circle containing $e_{3}$ and $f_{3}$ as $e_{1}$ and $e_{2}$. Denote by $k$ the point of $e_{3} c$ such that $\left|e_{3} k\right|=\Delta(R)$ and by $l$ the point of $f_{3} c$ such that $\left|f_{3} l\right|=\Delta(R)$. Since $e_{1}, e_{2}, e_{3} \in \operatorname{conv}\left\{e_{3}, f_{3}, k, l\right\}$, it is sufficient to show that conv $\left\{e_{3}, f_{3}, k, l\right\}$ may be covered by a disk of radius $\rho$. The triangle $e_{3} f_{3} k$ is contained in a quarter of a disk of thickness $\Delta(R)$, so by Lemma 1 it can be covered by a disk of radius $\rho$. What is more such a disk is unique thanks to Lemma 1 For the same reason the triangle $e_{3} f_{3} l$ can be covered by a disk of radius $\rho$ and such a disk is unique. It is easily seen that the disk is the same for both triangles. This disk covers conv $\left\{e_{3}, f_{3}, k, l\right\}$, which ends proof in this case.

Assume now that for any $i, j, k$ such that $\{i, j, k\}=\{1,2,3\}$ the points $e_{i}$ and $e_{j}$ lay in $L_{k}$ on the different sides of the great circle containing $e_{k}$ and $f_{k}$. Let us stay with this assumption up to the end of the proof.

Let us consider the case when the triangle $e_{1} e_{2} e_{3}$ is obtuse or right. Without losing the generality we can assume that the angle $\angle e_{1} e_{3} e_{2}$ is obtuse or right. Let $k$ be the point on the same side of the great circle containing $e_{1}$ and $e_{2}$ as $e_{3}$, such that $\left|e_{1} k\right|=\left|e_{2} k\right|$ and the angle $\angle e_{1} k e_{2}$ is right. By Theorem 8 of [7] $\left|e_{1} e_{2}\right|$ is at least $\arccos \left(\cos ^{2} \Delta(R)\right)$. Therefore $\left|e_{1} k\right|$ and $\left|e_{2} k\right|$ are at most $\Delta(R)$, because otherwise by (3) the lenght $\left|e_{1} e_{2}\right|$ is greater that $\arccos \left(\cos ^{2} \Delta(R)\right)$. Thus by Lemma 1 the circumradius of the triangle $e_{1} k e_{2}$ is at most $\arctan \left(\sqrt{2} \cdot \tan \frac{\Delta(R)}{2}\right)$.

By Lemma 6 we easily obtain that for any point $t$ from the circle circumscribed on $e_{1} k e_{2}$ laying on the same side of the great circle containing $e_{1}$ and $e_{2}$ as $k$, the angle $\angle e_{1} t e_{2}$ is at least $\frac{\pi}{2}$. Thus since $\angle e_{1} e_{3} e_{2}$ is obtuse or right, $e_{3}$ must lay inside this circumscribed circle, which ends the proof in this case.

The last case is when the triangle $e_{1} e_{2} e_{3}$ is acute. Clearly, all heights of this triangle have lengths less that $\Delta(R)$. Let $g$ be the point closest to $e_{2}$ such that $e_{2}$ is on the arc $e_{1} g$ and $g$ satisfies at least one of following conditions: $\angle e_{1} e_{3} g$ is right or $\operatorname{dist}\left(g, e_{1} e_{3}\right)=\Delta(R)$ or $\operatorname{dist}\left(e_{1}, g e_{3}\right)=\Delta(R)$. If $\angle e_{1} e_{3} g$ is right, then $\left|e_{1} e_{3}\right|$ and $\left|e_{3} g\right|$ are at most $\Delta(R)$. Therefore the triangle $e_{1} g e_{3}$ is contained in a right triangle with legs of length $\Delta(R)$, and so in the quarter of a disk of thickness $\Delta(R)$. Hence, by Lemma 1, $e_{1}, e_{2}, e_{3}$ can be covered by a disk of radius $\rho$.

Otherwise, the angle $\angle e_{1} e_{3} g$ is acute. Since $\left|\angle e_{1} g e_{3}\right|<\left|\angle e_{1} e_{2} e_{3}\right|$, the triangle $e_{1} g e_{3}$ is acute. One height of this triangle, say this from the vertex $g$, is of length $\Delta(R)$. Moreover, the height of this triangle from vertex $e_{1}$ is of length at most $\Delta(R)$. Let $j$ be the point closest to $e_{3}$ such that $e_{3}$ is on the arc $e_{1} j$ and $j$ satisfies at least one of following conditions: $\angle e_{1} g j$ is right or $\operatorname{dist}\left(j, e_{1} g\right)=\Delta(R)$ or $\operatorname{dist}\left(e_{1}, g j\right)=\Delta(R)$. If $\angle e_{1} g j$ is right (which by the 
way is possible only if $\left.\Delta(R)=\frac{\pi}{2}\right)$, then we apply the same argument as in the preceding case. Otherwise the triangle $e_{1} g j$ is acute and has two heights of length $\Delta(R)$, say from vertices $g, j$. Observe that from the construction of this triangle we obtain that the third height is of length at most $\Delta(R)$. Since the points $e_{1}, e_{2}, e_{3}$ are contained in the triangle $e_{1} g j$, it remains to prove that this triangle may be covered by a disk of radius $\rho$.

Observe that since the triangle $e_{1} g j$ has two equal heights, it is an isosceles triangle. Denote the angle at $e_{1}$ by $2 \alpha$, the center of the circle circumscribed on $e_{1} g j$ by $o$ and the radius of this circle by $\sigma$. Denote also the point at the middle of $j e_{1}$ by $k$ and the point on $e_{1} g$ closest to $j$ by $h$. Clearly, the triangles $h j e_{1}$ and $o k e_{1}$ are right. Put $B=\left|j e_{1}\right|$ and notice that $\left|k e_{1}\right|=\frac{B}{2}$. Since $e_{1} g j$ is isosceles, we have $\left|\angle o e_{1} k\right|=\alpha$. The length of the arc $h j$ is $\Delta(R)$.

From (11) for the triangle $o k e_{1}$ we obtain $\tan \frac{B}{2}=\cos \alpha \tan \sigma$. Formula (2) for the triangle $h j e_{1}$ gives $\sin \Delta(R)=\sin 2 \alpha \sin B$ and in different form $\sin B \cos \alpha=\frac{\sin \Delta(R)}{2 \sin \alpha}$. Using formulas from last two sentences and the trigonometric formula $\tan \frac{B}{2}=\frac{1-\cos B}{\sin B}$, we obtain $\tan \sigma=\frac{\tan \frac{B}{2}}{\cos \alpha}=\frac{1-\cos B}{\sin B \cos \alpha}=$ $\frac{1-\cos B}{\frac{\sin \Delta(R)}{2 \sin \alpha}}=\frac{2 \sin \alpha\left(1-\sqrt{1-\sin ^{2} B}\right)}{\sin \Delta(R)}=\frac{2 \sin \alpha\left(1-\sqrt{1-\frac{\sin ^{2} \Delta(R)}{\sin ^{2} 2 \alpha}}\right)}{\sin \Delta(R)}=$ $\frac{2\left(\sin \alpha-\sqrt{\sin ^{2} \alpha-\frac{\sin ^{2} \alpha \sin ^{2} \Delta(R)}{\sin ^{2} 2 \alpha}}\right)}{\sin \Delta(R)}=\frac{2\left(\sqrt{1-\cos ^{2} \alpha}-\sqrt{1-\cos ^{2} \alpha-\frac{\sin ^{2} \Delta(R)}{4 \cos ^{2} \alpha}}\right)}{\sin \Delta(R)}$.

The greatest possible value of $\alpha$ is $\frac{\pi}{4}$ and the smallest is this value for which the third height is of length $\Delta(R)$. In the first case the triangle $e_{1} j g$ is right and by Lemma 1 we have $\tan \sigma_{1}=\sqrt{2} \cdot \tan \frac{\Delta(R)}{2}$. By the formula $\tan \Delta(R)=\frac{2 \tan \frac{\Delta(R)}{2}}{1-\tan ^{2} \frac{\Delta(R)}{2}}$ we easily obtain that in this case

$$
\tan \sigma_{1}=\sqrt{2} \cdot \frac{\sqrt{4+4 \tan ^{2} \Delta(R)}-2}{2 \tan \Delta(R)}=\frac{\sqrt{2+2 \tan ^{2} \Delta(R)}-\sqrt{2}}{\tan \Delta(R)}
$$

In the second case the triangle $e_{1} j g$ is equilateral and by Lemma 3 in this case $\tan \sigma_{2}=\frac{\sqrt{9+8 \tan ^{2} \Delta(R)}-3}{2 \tan \Delta(R)}$. For shortness denote $\tan \Delta(R)=t$. We have $\tan \sigma_{2}=\frac{\sqrt{9+8 t^{2}}-3}{2 t}=\frac{1}{2 t}\left(\sqrt{9+8 t^{2}}-\sqrt{8+8 t^{2}}-3+\sqrt{8+8 t^{2}}\right)=$ $\frac{1}{2 t}\left(\frac{1}{\sqrt{9+8 t^{2}}+\sqrt{8+8 t^{2}}}-3+\sqrt{8+8 t^{2}}\right)<\frac{1}{2 t}\left(\frac{1}{\sqrt{9}+\sqrt{8}}-3+\sqrt{8+8 t^{2}}\right)=$ $\frac{1}{2 t}\left(3-2 \sqrt{2}-3+\sqrt{8+8 t^{2}}\right)=\frac{\sqrt{2+2 t^{2}}-\sqrt{2}}{t}=\tan \sigma_{1}$.

If we put $\cos ^{2} \alpha=x$ and $\frac{\sin ^{2} \Delta(R)}{4}=c$, then by Lemma 5 we conclude that $\tan \sigma$ has the greatest value $\max \left(\tan \sigma_{1}, \tan \sigma_{2}\right)=\sqrt{2} \cdot \tan \frac{\Delta(R)}{2}$, from which we obtain the thesis of theorem. 
Note that by Lemma 1 in general we can not improve the estimate from Theorem 2

\section{References}

[1] L. Danzer, B. Grünbaum, V. Klee, Helly's theorem and its relatives, in Proc. of Symp. in Pure Math. vol. VII, Convexity, (1963), pp. 99-180.

[2] B. V. Dekster, The Jung theorem for spherical and hyperbolic spaces, Acta Math. Hungar., 67 (4) (1995), 315-331

[3] H. Han, T. Nishimura, Self-dual Wulff shapes and spherical convex bodies of constant width $\frac{\pi}{2}$, J. Math. Soc. Japan, vol. 69, Number 4 (2017), 1475-1484.

[4] M. Lassak, On the smallest disk containing a planar reduced convex body, Arch. Math. 80 (2003), 553-560.

[5] M. Lassak, Width of spherical convex bodies, Aequationes Math., 89 (2015), no. $3,555-567$.

[6] M. Lassak, H. Martini, Reduced convex bodies in Euclidean space - a survey, Expositiones Math. 29 (2011), 204-219.

[7] M. Lassak, M. Musielak, Reduced spherical convex bodies, Bulletin of the Polish Academy of Sciences, to appear (see also arXiv:1607.00132 v1).

[8] M. Lassak, M. Musielak, Spherical bodies of constant width, Aequationes Math., to appear (see also: arXiv:1801.01161 v1).

[9] L. A. Masal'tsev, Incidence theorems in spaces of constant curvature, J. Math. Sci., Vol. 72 (1994), 3201-3206

[10] J. Molnár, Über einen Übertragung des Hellyschen Satzes in sphärische Räume, Acta Math. Acad. Sci. Hungary 8 (1957), 315-318

[11] D.A. Murray, Spherical Trigonometry, Longmans Green and CO, London, Bombay and Calcuta, (1900).

[12] T. Nishimura, Y. Sakemi, Topological aspect of Wulff shapes, J. Math. Soc. Japan., 66 (2014), 89-109.

Michał Musielak

University of Science and Technology

Kaliskiego 7, 85-789, Bydgoszcz, Poland

e-mail: michal.musielak@utp.edu.pl 